\title{
A foreign body lodged in the sub-mental space through to the retro-pharyngeal area: a review of anatomical risks and surgical approaches
}

\author{
Kaoutar Cherrabi* ${ }^{*}$, Zouheir Zaki and Mohamed Noureddine El Alami(D)
}

\begin{abstract}
Background: Foreign body inclusions are rare in head and neck. They are challenging in regard to anatomical risks and surgical approaches. This is the case of a particular foreign body with a curious trajectory, associated with a brief review of anatomical risks and surgical approaches.

Case presentation: This is the case of a 25 -year-old male who has been attacked with a sharp object, which caused an inclusion of a part of the foreign body in the sub-mental and pharyngeal areas.

The clinical exam showed a painful bulging in the sub-mental area, with moderately hemorrhagic sputum and difficulty while swallowing. The intra-oral exam showed a foreign body that was located at the right side of the base of the tongue, and which goes backwards and outwards to penetrate retro-pharyngeal mucosa.

The patients underwent an angio-CT scan, which showed a curious fine and sharp metallic object, without direct signs of lesions to the jugular vein or carotid artery or any collateral branches.

The patient underwent extraction through cervical approach, with satisfying bleeding control. Intra-oral exploration showed a retro-pharyngeal lesion of $2 \mathrm{~cm}$, without particular bleeding. The cervical lesion and retro-pharyngeal lesions were closed. The lesion at the base of the tongue was of $1 \mathrm{~cm}$, palpable but not accessible to sutures, and a naso-pharyngeal tube was inserted.

The patient presented very satisfying post-operative outcome, without any complications.

Conclusion: When dealing with foreign bodies of head and neck, physicians must be precocious and prepared for the risk of bleeding after extraction. Thorough radiological exploration is necessary as long as the patient is stable. Direct and indirect radiological signs allow the clinician to understand the nature and the trajectory of the object, as well as the damage to collateral structures.
\end{abstract}

Keywords: Foreign body, Cervical, Sub-mental, Sub-mandibular, Head and neck; Open wounds

${ }^{*}$ Correspondence: Cherrabi.kaoutar@gmail.com

ENT and Cervico-Facial Surgery Department, Fez, Morocco

\section{Background}

The presence of foreign bodies in head and neck traumatic lesions is rare, and they are associated with an elevated risk of complications, as well as a challenging management [1]. This is a rare case of a penetrating neck wound with a curious foreign body inclusion. The case presentation is associated with a review of the 
classification of neck injuries, a review of different surgical approaches depending on the nature of the lesions, and a discussion of radiological tools.

\section{Case presentation}

This is the case of a 25-year-old man that sustained an open wound to the sub-mental region, with the inclusion of an unknown foreign body.

The patient presented bloody sputum, a difficulty swallowing: signs of hemodynamic or neurological distress.

Clinical exam showed a neat entry wound, of $1 \mathrm{~cm}$ diameter, in the right side of sub-mental, $2 \mathrm{~cm}$ to the median line without active bleeding. Intra-oral exam showed a foreign body originating from the base of tongue to the retro-pharyngeal area, behind the posterior pillar of palatine region.

The patient did not have any history of medical of surgical conditions.

The patient was admitted $6 \mathrm{~h}$ after the injury, vital signs were monitored, and blood tests were immediately requested, and patient was prepared to be admitted to the operating room.

The patient underwent angio-CT scan, which showed a thin metallic object of a few millimeters diameter, without direct signs of injury to the carotid artery or internal jugular vein (Figs. 1 and 2), the nature of the object was difficult to deduce.

The patient was admitted to the operating room, antibioprophylaxis was administered as well as analgesia.

Under general anesthesia, the extraction of the object was performed through a cervical approach. A mini-Sebileau incision was performed, dissection of myo-cutaneous structures, careful dissection of the vascular space to prepare the approach to vascular structures in cases of heavy bleeding after mobilization of foreign body. After dissection of the sub-mandibular space, the object was lodged anterior to the medial border of the mandibular gland. The extraction of the foreign object was not followed by an active bleeding, careful hemostasis was carried out. The foreign body was a sharp pencil, broken in its the blunt end. The retro-pharyngeal lesion was closed, and a naso-pharyngeal tube was inserted (Figs. 3 and 4).

The post-operative follow-up of the patient was without incidents, the patient had satisfying naso-fibroscopy.

Complete tissue healing was obtained after 1 month follow-up.

\section{Discussion}

\section{Background}

Penetrating neck injury is defined as an injury that goes through the platysma muscle $[1,2]$. They can be life-threatening depending on their location and extension, especially since it is often adjacent to vascular,

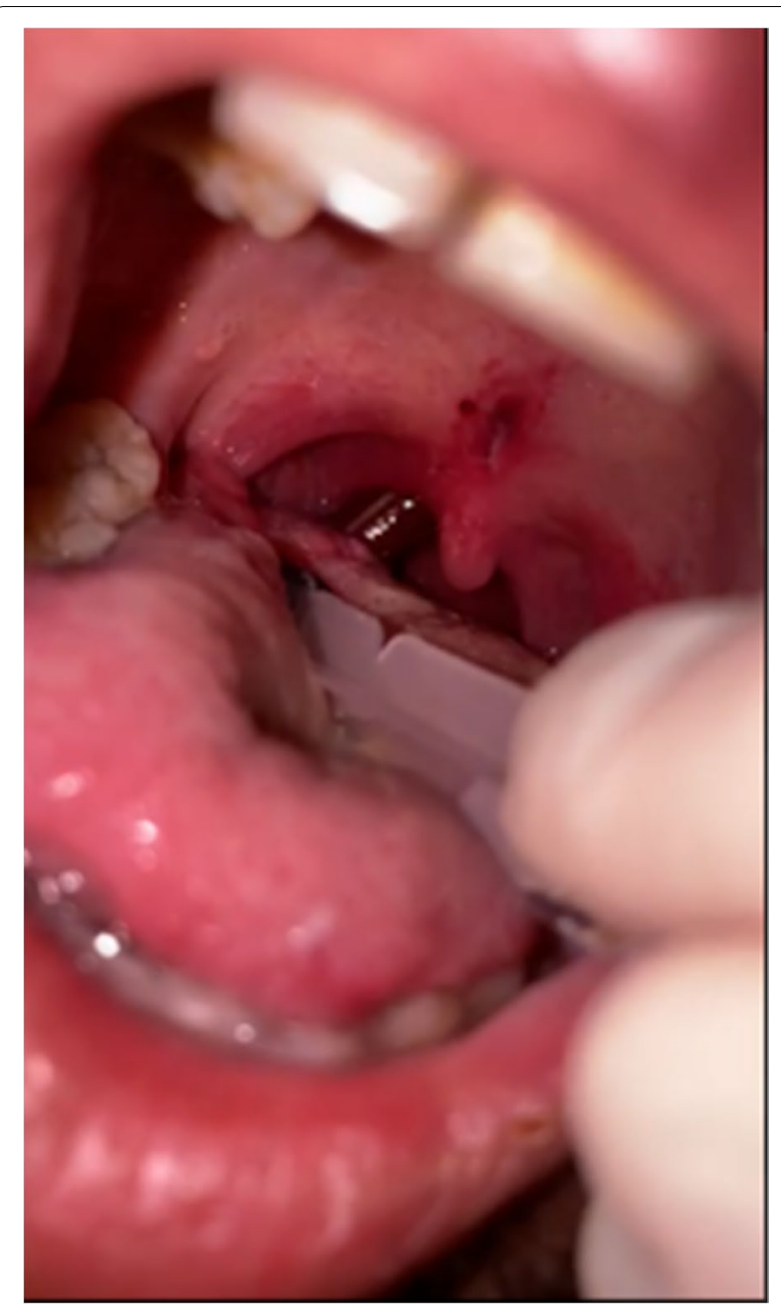

Fig. 1 Clinical aspect

neurological, and aero-digestive structures $[1,2]$. The classical zonal approach is progressively replaced by a hollistic approach of neck injuries [2].

- The causes of injury are various: occupational, selfinflicted (suicide attempt), domestic accidents, and assaults. Beside the mechanisms that are associated with more mortality such as blasts, gun and riffle shots, stabbing, and impalement $[1,3]$.

- The direction of kinetic force and the axis of weight transmission are two important factors in understanding the severity of the impact as well as the extent of the damage to surrounding structures [1].

Traditional approach to penetrating injuries of the neck were based on the external wound's location since the work of Monson et al. (1967), it describes three zones $[1,4-6]$. 


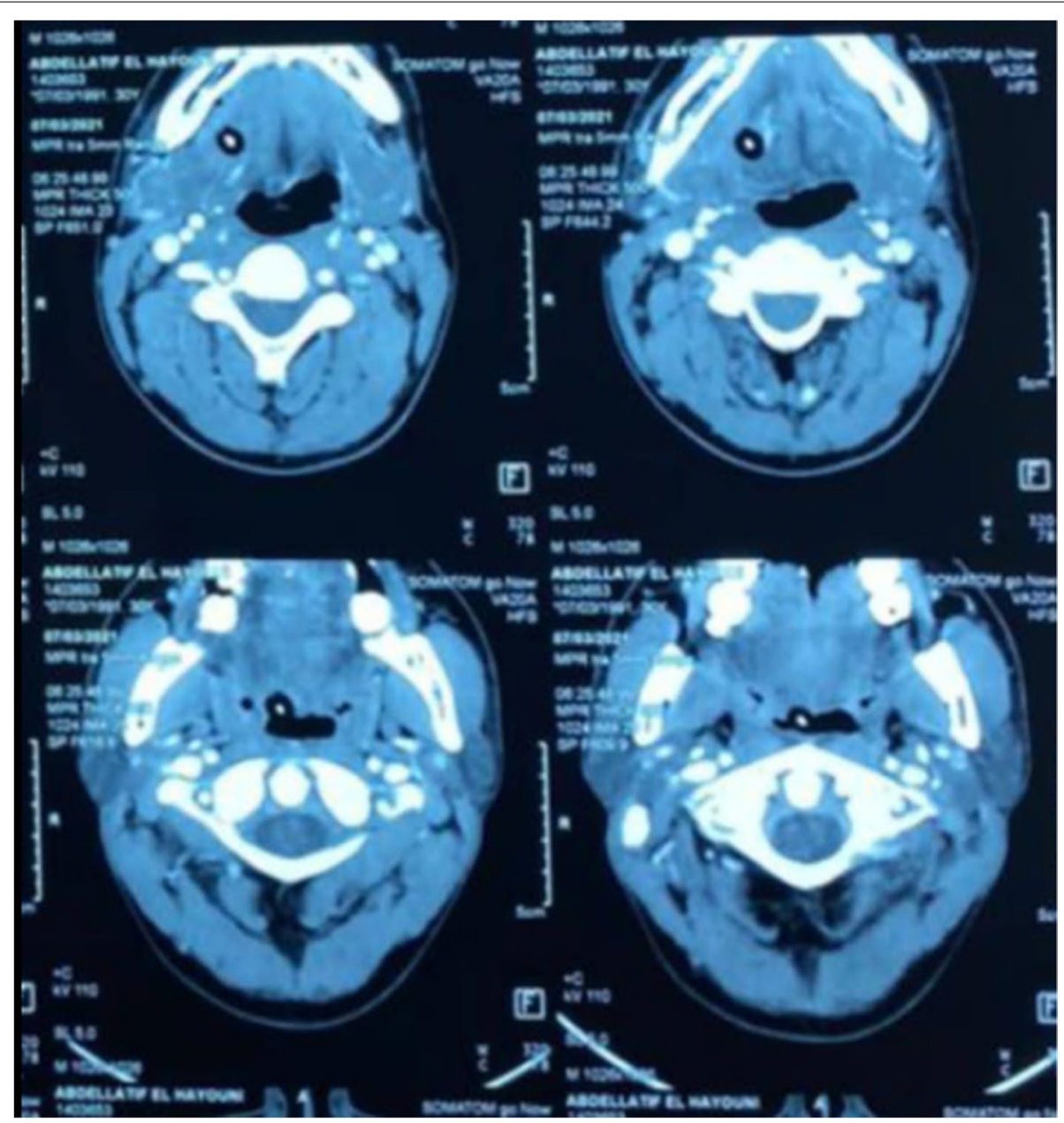

Fig. 2 Axial CT-scan section showing the trajectory of foreign body with a metallic component

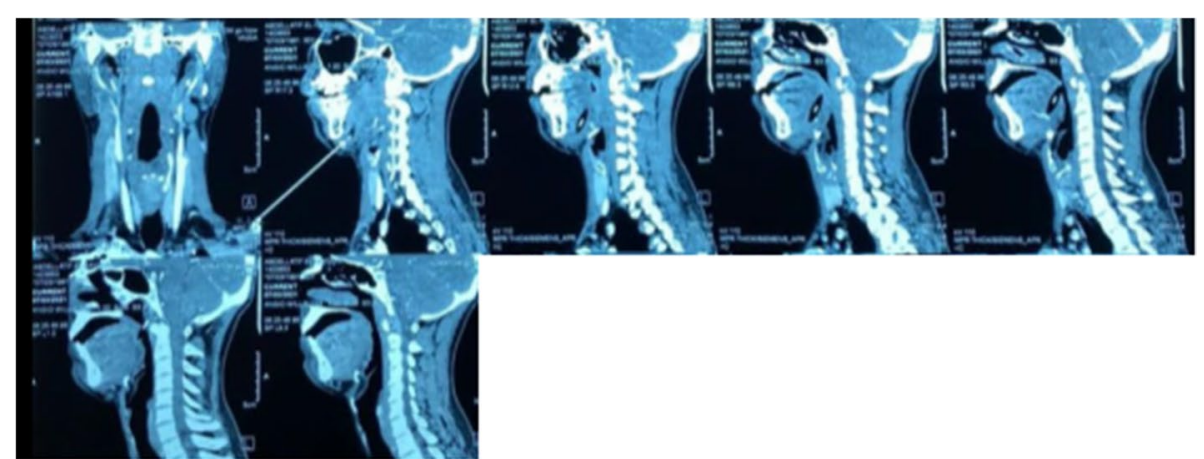

Fig. 3 Sagittal CT-scan section showing the trajectory of foreign body with a metallic component

Full medical history and clinical exam are very important factors whenever the patient is stable [1].

Diagnosis of neck injuries that require urgent general management and surgical treatment is quite challenging and generally complex. The morbidity associated with it depends on the severity of the underlying trauma [1]. Diagnosis of a remanent part of a foreign 
body is also difficult especially when the foreign body isn't fully visible [1].

- Hard signs are indications for immediate surgery for surgical exploration and repair [2, 6].

- The different radiological tools have each their own advantages and limitations [1]. Several radiological tools have been utilized to assess penetrating injuries to the neck, CT scan with or without enhancement, angio-MRI, Doppler sonography, and angiography [5].

Radiological assessment depends on the expected material of the foreign body (wood, glass, metal, tooth, debris), in order to have a minimal rate of negative findings $[1,5,7,8]$.

Preoperative assessment including the implementation of contrast enhanced CT scan could help visualize damage to surrounding vascular structures [1].

Multi-dimension computed tomography is most successful for detecting foreign bodies; however, it struggles with wooden objects $[1,5]$. Moreover, it reveals the integrity of aero-digestive tract, neuro-vascular structures, vertebrae, and the trajectory and often times the nature of the foreign body [4]. It has been progressively replacing MRI and angiography in diagnosis of neck injuries. ${ }^{8}$ According to Fergueson et al., in absence of hard signs of vascular injury, selective arterial angiography has sensibly the same false-positive cases rate as clinical evaluation [5]. Besides, MRI could cause re-location of metallic objects and is generally counter-indicated, as metallic foreign bodies are considered ferromagnetic until proven otherwise [5].

Unless associated with a wooden object, it is difficult to diagnose first hand; retained wooden objects are associated with a high rate of infection, which can be fatal in some cases [9]. Retain wooden object show increased radiological density, due to liquid retention, which enhances radiological diagnosis [9].

Optimal planning of removal of foreign body includes a thorough evaluation [1]. The traditional management of penetrating neck wound was zone-based since the work of Monson et al. in 1969. However, low et al. (2014) have established that there is no correlation between internal injuries and external wounds, the zone-based approach hinders the exploration of lesions in stable patients. Nowicki et al. [2] propose a non-zonal approach that considers the neck as a whole.

Wound cleaning to remove debris is important to prevent re-infection of the wound $[2,5]$. Cases of re-infected wounds should imply the possibility of a remanent foreign body $[1,5]$.

Post-operative medical therapy includes

"Antibiotherapy: Cefazolin 2 g every 8 h, or ampicillin associated with sulbactam $2 \mathrm{~g}$ every $6 \mathrm{~h}$ for $10-14$ days. It is indicated in the presence of clinical and or biological signs of infection [1]. Broad spectrum treatment, first generation cephalosporin, or clindamycin for empiric therapy.

For intra-oral wounds, antibacterial mouth rinse $0.12 \%$ chlorexidine [3].

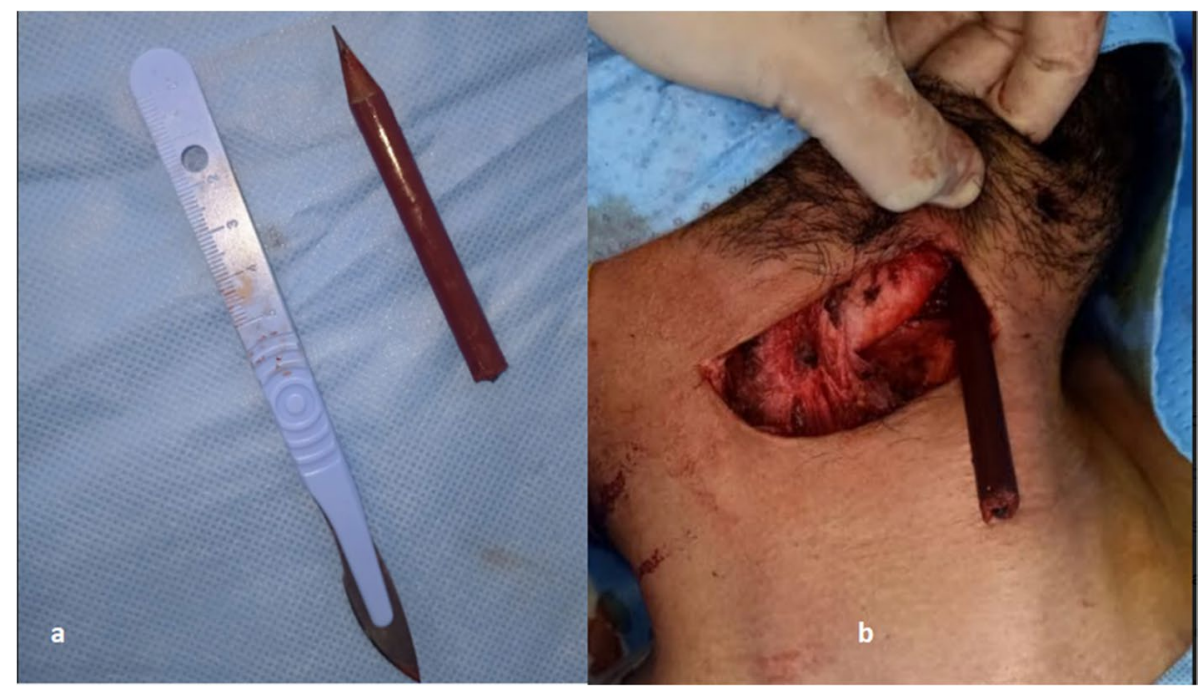

Fig. 4 Per-operative aspect. a Foreign Body extracted. b Pre-operative view 


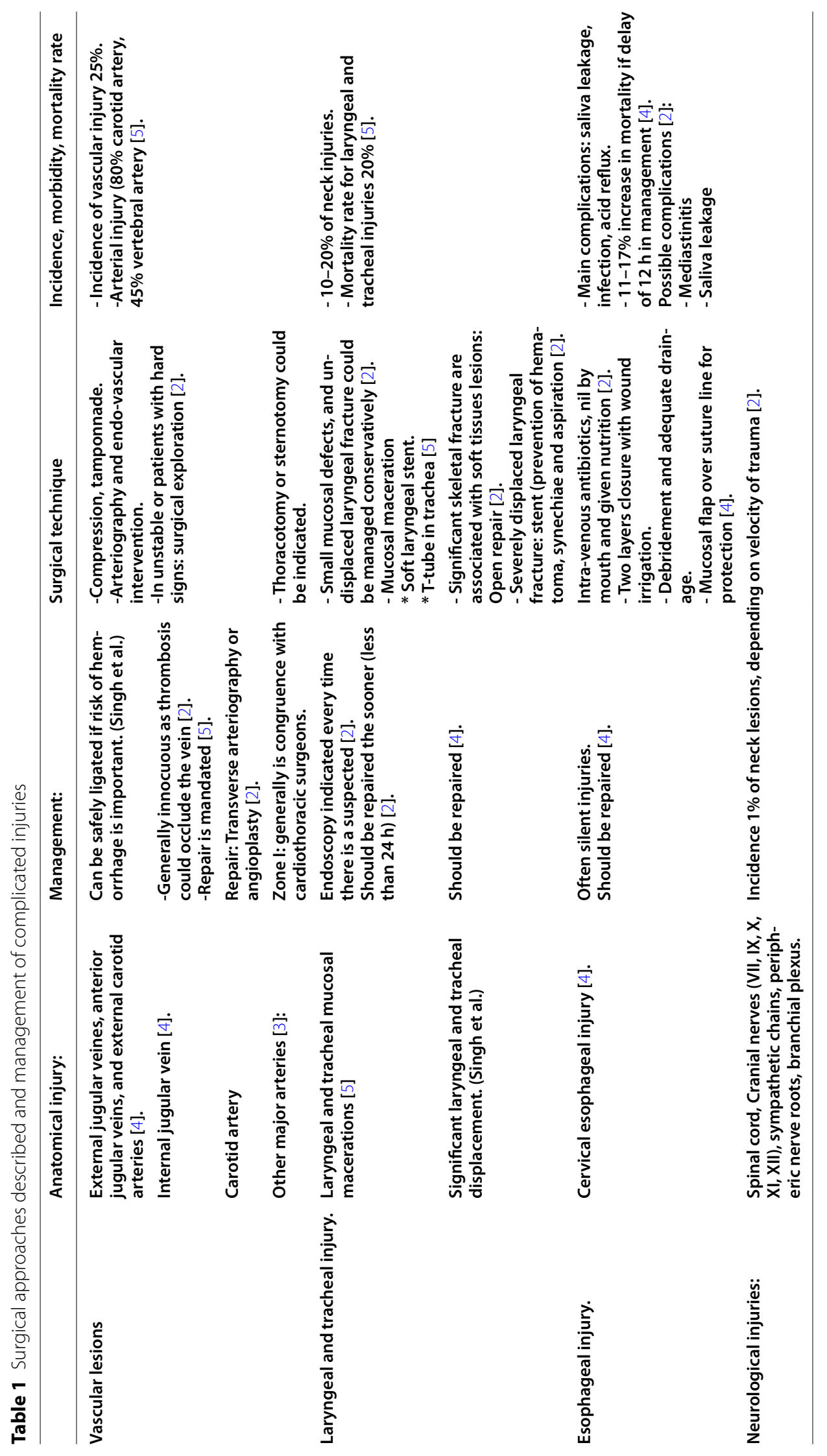


*Antibioprophylaxis depends on the delay in management and the nature of the penetrating object [1]. "Tetanos prophylaxis [1].

*Analgesics [5].

Decision for surgery depends on stability of patients and the presence of hard signs [2].

Incisions that are mostly used for exploration of neck injuries are Kocher incision, vertical cervical incision, supra-clavicular incision; sternotomy, and thoracotomy [7].

Surgical management of neck injuries is summarized in Table 1.

Possible complications: acute or chronic infection and immune response in reaction to foreign bodies. Neurological impairments, functional, mechanical, and esthetic impairment [5]. Mortality rate of head and neck injuries $3-6 \%[5,6,7,8]$.

\section{Conclusions}

This is the rare case of a foreign body inserted through a penetrating wound straddling cervical and-facial regions. Radiological exploration when the patient is stable is key to understanding the anatomical risks, and the surgical approach. Foreign bodies of head and neck are rare entities, and the physician must be precautious and prepared for the risk of bleeding after extraction, thorough radiological exploration is necessary as long as the patient is stable.

\section{Abbreviations}

CT scan: Computed tomography; MRI: Magnetic radiological imaging.

\section{Acknowledgements}

Not applicable.

\section{Authors' contributions}

KC conceived and designed the analysis, collected the data, performed the analysis, and wrote the paper. ZZ contributed data or analysis tools. MNE contributed data or analysis tools. All authors have read and agreed to its content.

\section{Funding}

Not applicable.
Availability of data and materials

Not applicable.

\section{Declarations}

Ethics approval and consent to participate

Not applicable.

\section{Consent for publication}

The subject provided written consent for participation and publication of case report.

\section{Competing interests}

The authors have no conflict of interest to declare.

Received: 20 April 2021 Accepted: 1 November 2021

Published online: 17 November 2021

\section{References}

1. Voss J, Thieme N, Doll C et al (2018) Penetrating foreign bodies in head and neck trauma: a surgical challenge. Craniomaxillofac Trauma Reconstr 11(3):172-182. https://doi.org/10.1055/s-0038-1642035

2. Nowicki JL, Stew B, Ooi E (2018) Penetrating neck injuries: a guide to evaluation and management. Ann R Coll Surg Engl 100(1):6-11. https://doi.org/10.1308/rcsann.2017.0191 Epub 2017 Oct 19. PMID: 29046084; PMCID: PMC5849205

3. Nashef R, Dodson TB (2017) Penetrating Injuries of the Face. In: Penetrating Trauma. Springer, Berlin, Heidelberg, pp 221-232

4. Singh RK, Bhandary S, Karki P (2009) Managing a wooden foreign body in the neck. J Emerg Trauma Shock 2(3):191-195. https://doi.org/10. 4103/0974-2700.55340 PMID: 20009310; PMCID: PMC2776368

5. Voss $\mathrm{JO}$ et al (2021) Imaging foreign bodies in head and neck trauma: a pictorial review. Insights Imaging 12(1):1-16

6. Steenburg SD, Leatherwood D (2016) Penetrating neck trauma: a review of image-based evaluation and management

7. Bagheri SC (2008) H. Ali Khan, and R. Bryan Bell. Penetrating neck injuries. Oral Maxillofac Surg Clin North Am 20(3):393-414

8. NASR Adonis et al. (2015) Evaluation of the use of tomography in penetrating neck trauma. Rev Col Bras Cir. 42; 215-219. https://doi.org/10. 1590/0100-69912015004004. n.4 [cited 2021-04-14], ISSN 1809-4546.

9. Krimmel M, Cornelius CP, Stojadinovic S, Hoffmann J, Reinert S (2001) Wooden foreign bodies in facial injury: a radiological pitfall. Int J Oral Maxillofac Surg 30(5):445-447. https://doi.org/10.1054/ijom.2001.0109 PMID: 11720049

\section{Publisher's Note}

Springer Nature remains neutral with regard to jurisdictional claims in published maps and institutional affiliations.

\section{Submit your manuscript to a SpringerOpen ${ }^{\circ}$ journal and benefit from:}

- Convenient online submission

- Rigorous peer review

- Open access: articles freely available online

- High visibility within the field

- Retaining the copyright to your article

Submit your next manuscript at springeropen.com 